\title{
Initial Results of Oral Thrombolytic Agent Clinical Application ${ }^{\dagger}$
}

\author{
Pavel Madonov ${ }^{1, *}$, Stanialav Leont'ev ${ }^{2}$, Sergey Zotov ${ }^{3}$, Maksim Ufimtsev ${ }^{3}$, \\ Svetlana Mishenina ${ }^{1}$ and Dmitrii Kinsht ${ }^{1}$ \\ 1 Department of Pharmacology, Pharmaceutical Faculty, Novosibirsk State Medical University, 630091 \\ Novosibirsk, Russia; m-svetlana@ngs.ru (S.M.); kinsht@scpb.ru (D.K.) \\ 2 Pirogov Russian National Research Medical University, Faculty of Medicine, 117997 Moscow, Russia; \\ leontyevsg@bk.ru \\ 3 South Ural State Medical University, Faculty of Medicine, 454092 Chelyabinsk, Russia; \\ spzotov@mail.ru (S.Z.); dokm@list.ru (M.U.) \\ * Correspondence: pmadonov@yandex.ru; Tel.: +7-913-379-8303 \\ + Presented at the 25th Biennial International Congress on Thrombosis, Venice, Italy, 23-26 May 2018. \\ Published: 20 July 2018
}

\begin{abstract}
At the moment, there is no oral thrombolytic drug on the world pharmaceutical market. The drug Trombovazim, which has a thrombolytic effect under oral administration, has been registered in Russia. It differs from all thrombolytics - it has a fundamentally different mechanism of action. It is not an activator of plasminogen. It independently dissolves a thrombus. It is a direct thrombolytic. The article presents the materials of the initial studies of the drug Trombovazim.
\end{abstract}

Keywords: thrombosis of the venous system; thrombolytic therapy; oral thrombolytic

\section{Introduction}

Standard therapy for deep vein thrombosis of the lower extremities includes anticoagulants and antiaggregants, such as bacterial plasminogen activators (streptokinase, staphylokinase), tissue plasminogen activators (ateplase, tenecteplase, purolase, urokinase, etc.), plasmin (humanfibrinolysinum, and in project genetic engineered). Dangerous complications seriously limit the use of thrombolytic plasminogen activators. We have developed an oral thrombolytic Trombovazim, wich is exogenous thrombolytic proteinase (subtilisin), immobilized on polyethylene glycol, and present the main results of the study on the efficacy and safety of lower limbs deep vein thrombosis treatment with the inclusion of this thrombolytic in therapy.

\section{Objective}

The objective of this study is to analyze the efficacy and safety of lower limbs deep vein thrombosis treatment with the inclusion of an oral thrombolytic drug based on immobilized subtilisin (Trombovazim ${ }^{\circledR}$ ) in therapy.

Study tasks:

1. Evaluation of the thrombolytic effect of 3 different drug doses after a 12-day course of treatment of patients with a visualized thrombus of lower limbs deep vein.

2. Evaluation of the revascularization in the zone of compromised blood flow after course of treatment with three different doses of the drug.

3. Determination of the most effective drug dose.

4. Assessment of the drug tolerance and safety. 


\section{Methods}

A multicenter, randomized, double-blind, placebo-controlled clinical trial.

154 volunteers participated in the clinical trial «VETTER-1». All of them received only conservative standard therapy, including anticoagulants (low molecular weight heparins, unfractionated heparins, vitamin $\mathrm{K}$ antagonists), nonsteroidal anti-inflammatory drugs and phlebotrophic drugs. None of the participants underwent surgical intervention. The mode of mechanical compression was not taken into account.

All participants were divided into 2 groups $-\operatorname{control}(n=59)$ and observation $(n=95)$ group. The control group received a placebo in the form of tested drug with an inactivated substance. The observation group was divided into 3 subgroups consisting of 28, 32 and 35 participants who received tested drug at a dose of 1600, 3200 and 4800 U/day, respectively. The groups were comparable of analyzed characteristics: age, anthropometric statistics, gender, number of days from the disease, localization of thrombus (tibia, femur, tibia + femur), number of visualized thrombus, and laboratory analysis data before initiation of therapy.

The drug effectiveness was determined by means of an objective control method-blood flow ultrasound angioscanning, which reveals presence of a thrombus in thrombosed vein and completeness of revascularization. Statistical treatment of the results was carried out after trial completion.

\section{Results}

Comparison of revascularization in the zone of compromised blood flow revealed a distinct dose-dependent effect: subgroups that received drug at a dose of 1600, 3200 and $4800 \mathrm{U} /$ day showed a relative frequency of positive dynamics of $0.7070(p=0.0535, n=28), 0.7257(p=0.0188, n=32)$ and $0.7470(p=0.0082, n=35)$ respectively, that clearly indicate an increase in the effectiveness of therapy by $21 \%, 24 \%$ and $28 \%$ (respectively). The study did not reveal any negative dynamics in the observation group, and recorded 3 such cases in the control group. The difference between frequency of thrombus lysis in the observation group was 0.87 versus 0.63 in the control group, but was statistically unreliable $(p=0.07)$. It should be noted that all participants who received tested drug at a dose of $4800 \mathrm{U} /$ day had complete dissolution of thrombus. The safety assessment was based on the analysis of vital functions, laboratory and instrumental examination of subgroup receiving the maximum drug dosage, and did not reveal any undesirable events at all visits during trials.

\section{Discussion}

Lower limbs veins thrombosis is a known and widespread problem. All drugs used in standard therapy affect the hemostatic system and have serious side effects. Trombovazim is only existed oral thrombolytic in the world for the time being. An active element of this medicine is an enzyme subtilyzin. It is not a plasminogen activator or plasmin, in fact, it is a proteinase-subtilyzin with a direct fibrinolytic action. Thrombovazim is an oral thrombolytic because its subtilisin is immobilized on the polymer by means of unique electron-beam technology (axis-technology), which is described in detail in [1]. In short, the chemical side of this process is that the electron stream creates radical groups on the polymer, and the active group of the polymer is absorbed on the enzyme. As a result, an enzyme globule is covered with a polymer-clad shell. But an active enzyme site functions thoroughly. Axis-technology provides high enteral absorption and bioavailability at the rate of $18 \%$ [2].

The direct thrombolytic effect has been proven in vitro. There were taken a blood sampling from the animals' caudal veins into Eppendorf test-tubes, where blood clot formations were for two and twenty-four hours. The formed blood clots with a mass of $0.4 \mathrm{~g}$ were separated from the plasma and removed to a glass container connected to a perfusomate. The weight of a fresh 2-h blood clot has decreased in 8 times for the period of $90 \mathrm{~min}$, The weight of an old 24-h blood clot has also decreased in 8 times for the period of two hours [3] (Figures 1 and 2). It is important that the dissolution of the thrombus was done layer by layer, without detachment of pieces of the thrombus. 


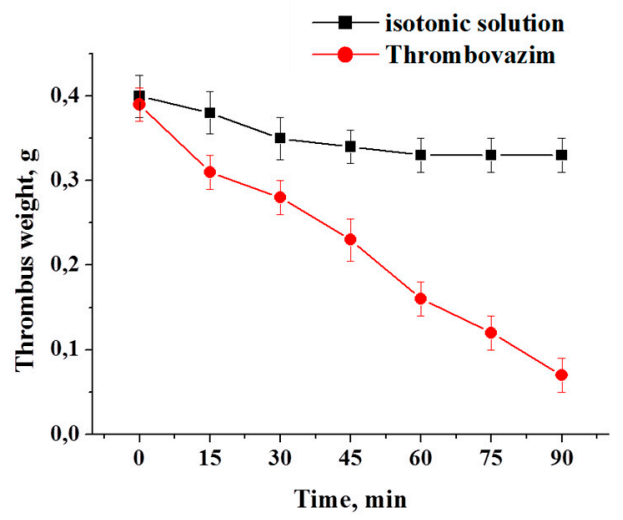

Figure 1. Dissolution of a 2-h thrombus.

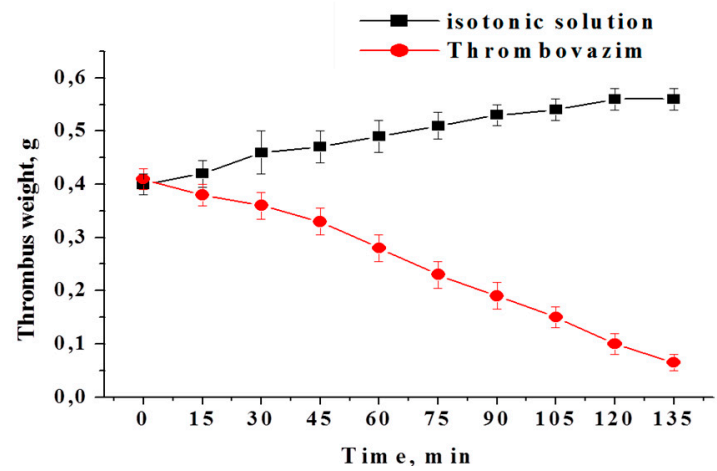

Figure 2. Dissolution of a 24-h thrombus.

A rat model of caudal veins thrombosis induced by k-carraginana showed that manifest thrombosis occurs in $12 \mathrm{~h}$ after the injection. Thrombosis has been eliminated in $36 \mathrm{~h}$ after the administration of Trombovazim per os [3] (Figure 3).

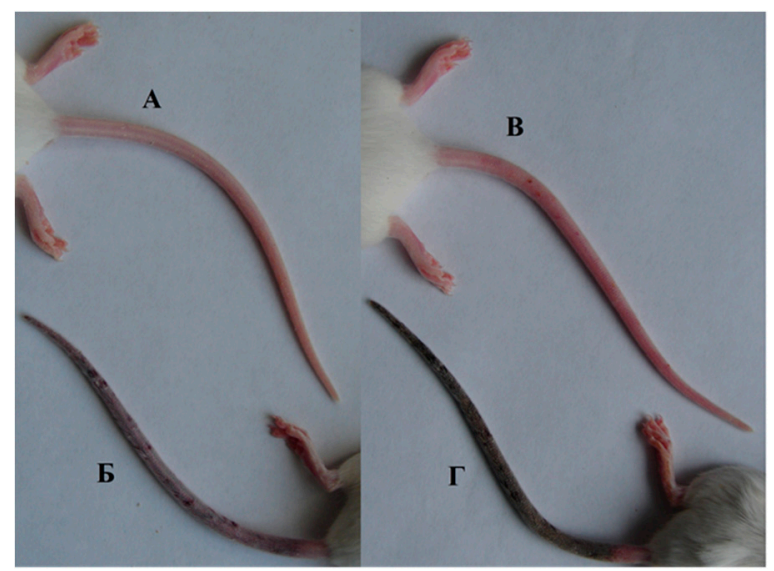

Figure 3. The rat model of tail veins thrombosis, induced by k-carraginan: A. Intact animals; Б. Animals $12 \mathrm{~h}$ after subcutaneous administration of k-carrageenan; B. Animals after $36 \mathrm{~h}$ after oral administration of Trombovazim; $\Gamma$. Animals without treatment, after $36 \mathrm{~h}$.

All these promising results gave hope that the drug will be effective in humans. Multicenter, randomized, placebo-controlled, double-blind clinical trial of the drug Trombovazim "Therapy of venous thrombosis VETTER-1" confirmed these assumptions. This is the first clinical research of the deep thrombosis of the lower extremities. The study was conducted for 3.5 years and 154 volunteers took part in the trial-men and women aged 35 to 75 years, with the presence of venous thrombosis in the lower extremities. All thrombosis was confirmed by ultrasound with duplex blood flow 
scanning. Duration of a disease: midsegment is 7 days, interquartile range-from 5 to $12-13$ days, range-from 2 to 37 days. There were 2 patient groups: placebo and study. Everybody was cured with a standard anticoagulant therapy, and in the study group, patients additionally received three doses of Trombovazim: 1600, 3200, 4800 units per day.

The main criterion of efficiency was the bloodstream recovery, recanalization, lysis of the floating part of the clot, bloodstream increase more than $5 \%$. Effectiveness evaluation was conducted on 10th day of therapy. Studies have shown that standard anticoagulation therapy is efficient in a half of the episodes, while taking Trombovazim in a dose of 4800 units per day to ensure the effectiveness of thrombolysis in $75 \%$ of episodes. A lysis of the floating part of the clot has been detected with a Trombovazim dose of 4800 units per day in all episodes, but it is not statistically reliable by reason of a small sample.

A pilot study of the efficacy of Trombovazim in the treatment of venous sinus thrombosis (unpublished data) also confirmed the efficacy of Trombovazim: when receiving a Trombovazim at a dose of 2400 units per day for 14 days in patients with tomographically visualized thrombus of venous sinus in $69 \%$ of cases there is no thrombus, in $46 \%$-there is a complete restoration of blood flow, and in $54 \%$-there is an increase in blood flow through the reduction of thrombus.

\section{Conclusions}

The use of the oral thrombolytic agent at a dose of 3200 and $4800 \mathrm{U} /$ day statistically significantly increases the effectiveness of lower limbs deep vein thrombosis treatment. The tested drug doses does not cause hemorrhagic complications and is well tolerated by patients. Analysis of the efficacy and safety of 3 different doses allows to consider $4800 \mathrm{U} /$ day the optimal dosage.

Author Contributions: P.M. conceived and designed the experiments and clinical trial, conducted a clinical trial, analyzed the data, wrote the paper; S.L. conducted a clinical trial, analyzed the data; S.Z. conducted a clinical trial, analyzed the data; M.U. conducted a clinical trial, analyzed the data; S.M. conducted experiments; D.K. conducted a clinical trial, analyzed the data.

Acknowledgments: The work was carried out with the financial support of Scientific Future Management, Novosibirsk, Russia.

Conflicts of Interest: The work was carried out with the financial support of Scientific Future Management (Novosibirsk, Russia). The founding sponsors had no role in the design of the study; in the collection, analyses, or interpretation of data; in the writing of the manuscript, and in the decision to publish the results.

\section{References}

1. Dygai, A.M.; Artamonov, A.V.; Bekarev, A.A.; Zdanov, V.V.; Zuzkov, G.N.; Madonov, P.G.; Udut, V.V. Nanotechnologies in Pharmacology; Publishing house RAMS: Moscow, Russia, 2011.

2. Vereshchagin, E.I.; Kinsht, D.N.; Madonov, P.G.; Dygai, A.M.; Plotnikov, M.B. Pharmacokinetics, antithrombotic and thrombolytic effect of a new oral drug Trombovazim. In Materials of XVI Russian Congress "Man and medicine", Proceeding of the XVI Russian Congress "Man and medicine", Moscow, Russian, 7-10 April 2009; Springer: Berlin, Germany, 2009; p. 629.

3. Mishenina, S.V.; Yershov, K.I.; Madonov, P.G.; Baykalov, G.I. Thrombolitic effects of subtilyzine drug on experimental models. Sib. Sci. Med. J. 2017, 37, 27-31. Available online: http://sibmed.net/article.php?lang=eng\&id_article=521 (accessed on 31 May 2018).

(C) 2018 by the authors. Licensee MDPI, Basel, Switzerland. This article is an open access article distributed under the terms and conditions of the Creative Commons Attribution (CC BY) license (http://creativecommons.org/licenses/by/4.0/). 